\title{
Design of Solar Powered and Fatigue Durable Quadcopter
}

\author{
Ying Xu*, Qianfeng Wan, Mwanza Taipa Raymond, Gang Lu, Xianghu Zeng \\ School of Aircraft Engineering, Nanchang Hangkong University, Nanchang, China \\ Email address: \\ tn416ky@126.com (Ying Xu),1600249323@qq.com (Qianfeng Wan),252336375@qq.com (M. T. Raymond), \\ 1811894355@qq.com (Gang Lu),1243806627@qq.com (Xianghu Zeng) \\ ${ }^{*}$ Corresponding author
}

\section{To cite this article:}

Ying Xu, Qianfeng Wan, Mwanza Taipa Raymond, Gang Lu, Xianghu Zeng. Design of Solar Powered and Fatigue Durable Quadcopter. International Journal of Mechanical Engineering and Applications. Vol. 7, No. 2, 2019, pp. 58-65. doi: 10.11648/j.ijmea.20190702.14

Received: April 22, 2019; Accepted: May 26, 2019; Published: June 15, 2019

\begin{abstract}
This paper features a new concept design of mini-quadcopter consisting of two types of power source systems. The solar type of power source and rechargeable lithium battery type of power source systems. The quadcopter design introduced showcases the extensive usefulness of solar cells on aircrafts. The title is "SPFD quadcopter", the SPFD stand for "solar powered fatigue durable" quadcopter. the quadcopter in question is designed to be powered by 40 solar thin film cells rated at $1.5 \mathrm{v}$ per panel. A fatigue analysis is conducted in its arm structures that offer support to the four mounted brushless motors responsible for driving the propellers that produce sufficient thrust to provide lift. By use of the "ANSYS" designing and simulating program, the carried out analysis shows the level of deformation in the arms due to the weight of the entire craft which tends to rest on the arms when the quadcopter is in flight. A fatigue structure analysis was considered necessary, to take appropriate precautions in both early stages of design and choice of material, to prevent premature structure failure in the field. Our objective of this paper is generally to show case the use of flexible solar cells as the source of power for providing power to the motors with the capability of charging the lithium battery during flight when being operated in battery mode. this new type of UAV can be used for military and civilian needs, surveillance and even space operations such as being set as a satellite to provide signal for internet back to earth.
\end{abstract}

Keywords: Quadcopter, Solar Power, Design, Fatigue Analysis

\section{Introduction}

UAVs have apparently proved to be of great benefit to the human race by their outstanding ability of carrying out operations in various conditions considered risky or hazardous to humans, one of their many advantages is the ability to fly over and through areas feared to possess high levels of radiation in search of survivors. For the SPFD case, pesticide daytime spraying in places of abundant sunlight like Africa, Zambia would be of great usefulness in the agricultural sectors of such countries that happen to possess higher sunlight intensities [1-2].

This paper features a new concept design of mini-quadcopter with an integrated dynamic power path management feature which simultaneously powers the system and charges the battery via solar energy. The quadcopter in question is designed to be powered by 36 solar thin film cells rated at $1.5 \mathrm{v}$ per panel. A fatigue analysis is conducted in its solar rib frame sections arm frame structures that offer support to the four mounted brushless motors which drive the propellers responsible for producing sufficient thrust to provide lift. By use of the "ANSYS" designing and simulating program, the carried out analysis shows the level of deformation in the arms due to the weight of the entire craft which tends to rest on the arms when the quadcopter is in flight. A fatigue structure analysis was considered necessary, to take appropriate precautions in both early stages of design and choice of material, to prevent premature structure failure in the field.

\section{Dynamic of Quadcopter}

\subsection{Design Objective of Device}

Our objective was to design and manufacture a quadcopter structure using the Ansys design modeler program. A device that would serve as multi-purpose in the field, as stated in the 
abstract, the main objective is to implement the use of solar cells as one of its sources of power and also the prospects of making such a device autonomous are considered.

Thus to say the design offers a provision for adjustments to such a robot to suit the conditions in the field. That is to say the operator will be capable of the following [2]:

(1) Make use of the solar cells during a sunny day.

(2) Isolate the solar frame and attached solar cells with ease.

(3) Handle $3-5 \mathrm{~kg}$ of pesticide.

(4) Long flight durations due to the installed MPPT [2] mechanism.

\subsection{Quad Copter Coordinate Systems}

This paper is about a quadcopter, a conceptual design of a new species of such a device whose main objective is to implement the use of solar cells as its source of power and also the prospects of making such a device autonomous are considered as shown in Figure 1 [2].

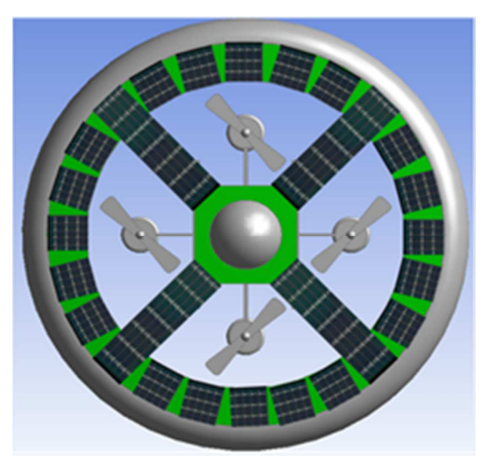

Figure 1. SPFD quadcopter

There exists two different coordinate systems when the quad copter is navigating in three dimensional space, the first one is mostly affected by motors which is the body coordinate system (shown in Figure 2 (a)), the second one happens to be the navigation frame (n), affected mostly by forces such as the gravitational forces [3]. Thus having two frames in which will operate, the first being the inertial frame which is defined by the ground with gravity pointing in the negative $\mathrm{z}$ direction as shown in Figure 2 (b). The second being the body frame which is defined by the orientation of the quadcopter with the rotor axes pointing in the positive $\mathrm{z}$ direction and the arms pointing in the $\mathrm{x}$ and $\mathrm{y}$ directions.

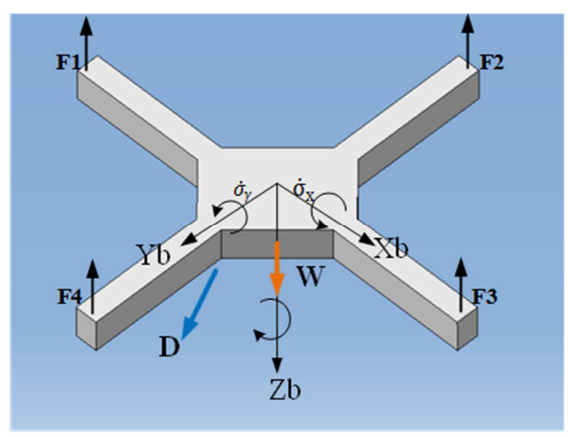

(a)

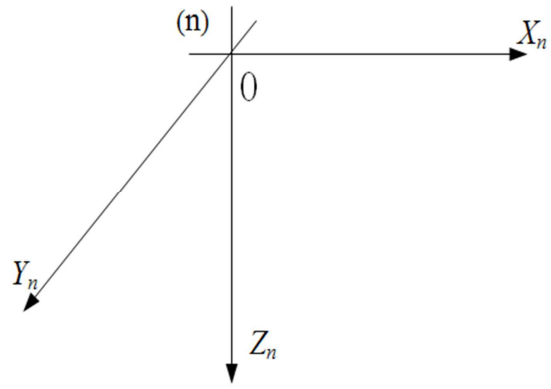

(b)

Figure 2. (a) Body reference frame; (b) Inertial reference frame.

Figure 2 (a) shows the forces acting on the quadrotor, thus the gravity, aerodynamic drag and the four thrust forces produced by the propellers also not forgetting two reference frames, thus the initial reference frame $(n)$ centered at 0 with unit vectors $x_{n}, y_{n}, z_{n}$, and the body reference frame (Figure 2 (a)) centered at $G$ with unit vectors. Also shown is the inertial orientation of the body frame.

The body coordinate system moves along with the quad copter, while the navigation coordinate system is the reference point of the quad copter. The quad copter is capable of rotating around its own axes with an angular velocity as $\dot{\sigma}$ of a corresponding axis [3-5].

\subsection{Motor Torque Calculation}

\subsubsection{Working Resistance Calculation}

The torque produced by the electric motors is given by:

$$
Q=K_{t}\left(I-I_{0}\right)
$$

Note: $Q$ is the motor torque; $I$ is the input current; $I_{0}$ is the no load current; $K_{t}$ is the torque constant [4].

We now consider the voltage across the motor which is given as:

$$
V=I R_{m}+K_{v} \omega
$$

Note: $V$ is the voltage drop across the motor; $R_{m}$ is the motor resistance in ohms; $\omega$ is the angular velocity of the motor; $K_{v}$ is RPM per volt.

Thus having the voltage across the motor as the sum of the back EMF and resistive loss, whereby we can now use this description to calculate the power consumed by the motors given as [6]

$$
P=\left(\frac{\left(Q+K_{t} I_{0}\right)\left(K_{t} I_{0} R_{m}+Q R_{m}+K_{t} K_{v} \omega\right)}{K_{t}^{2}}\right)
$$

Now we can assume a negligible motor resistance, also we take an assumption that $K_{t} I_{0}$ is much less than $Q$, furthermore, of which in practice this stands to be true since $I_{0}$ is the current with no load. Finally arriving at a more simplified expression for power given as:

$$
\mathrm{P} \approx \frac{\mathrm{K}_{\mathrm{v}}}{\mathrm{K}_{\mathrm{t}}} Q \omega
$$

\subsubsection{Effective Thrust Calculation}

Assuming that the free stream velocity is zero, such that air 
in the surrounding environment so happens to be stationary relative to the quadcopter $[3,7]$. By means of momentum theory, the equation of hover velocity as a function thrust is given as:

$$
v_{h}=\sqrt{\frac{T}{2 \rho A}}
$$

Note: $v_{h}$ is the hover velocity; $\rho$ is the density of the surrounding air; A is the swept out area by the motor.

By means of using the simplified power equation, we can therefore write as:

$$
P \approx \frac{K_{v}}{K_{t}} Q \omega=\frac{K_{v} K_{Q}}{K_{t}} T \omega=\frac{T^{\frac{3}{2}}}{\sqrt{2 \rho A}}
$$

Such that we now solve for the thrust magnitude $T$ and obtain a result expressing that thrust is proportional to the motors' angular velocity squared.

$$
T=\left(\frac{K_{v} K_{Q} \sqrt{2 \rho A}}{K_{t}} \omega\right)^{2}=k \omega^{2}
$$

Note: $\mathrm{k}$ is a constant; $\mathrm{T}$ is the thrust of a single motor.

Adding up the motors, total thrust on the quadcopter, thus in the body frame is given as [8]:

$$
\mathrm{T}_{\mathrm{b}}=\sum_{\mathrm{i}=1}^{4} \mathrm{~T}_{\mathrm{i}}=\mathrm{k}\left[\begin{array}{c}
0 \\
0 \\
\sum \omega_{\mathrm{i}}^{2}
\end{array}\right]
$$

\subsubsection{Frictional Resistance Calculation}

Total drag in all directions as:

$$
F_{D}=\left[\begin{array}{l}
-k_{d} \dot{x} \\
-k_{d} \dot{y} \\
-k_{d} \dot{z}
\end{array}\right]
$$

Note: $F_{D}$ is the overal drag force; $k_{d}$ is the drag constant.

Having solved for forces acting on the quadcopter, now we can consider solving for the torques. The torque under consideration is the torque required to keep the propeller spinning and is capable to continuously supply the quadcopter with sufficient thrust. This torque overcomes the frictional drag force and creates an instantaneous angular acceleration. From the fluid dynamics drag equation, frictional force is given as $[2,9]$ :

$$
F_{D}=\frac{1}{2} \rho C_{D} A v^{2}
$$

Note: $\rho$ is the surrounding fluid density; $A$ is the propeller cross-section area; $C_{D}$ is the dimensionless constant.

\subsubsection{Resistance Torque Calculation}

Such that the torque due to drag is given as:

$$
Q_{D}=\frac{1}{2} R \rho C_{D} A v^{2}=\frac{1}{2} R \rho C_{D} A(Q R)^{2}=b Q^{2}
$$

Note: $R$ is the radius of the propeller; $Q$ is the angular velocity of the propeller; $b$ is the dimensioned constant.

\section{Motion Equations}

The quadcopter is capable of achieving acceleration levels in the inertial frame, due to gravity, linear friction and of course thrust. By means of linear motion, we are capable of obtaining the thrust vector via the use of the rotation matrix $R$ in the inertial frame to map the thrust vector from the body frame to the inertial frame as shown in the given expression:

$$
m \vec{x}=\left[\begin{array}{c}
0 \\
0 \\
-m g
\end{array}\right]+R T_{B}+F_{D}
$$

Note: $\vec{x}$ is the position of the quadcopter; $g$ is the acceleration caused by gravity; $T_{B}$ is the thrust vector in the body frame.

Now we consider Euler's equations for rigid body dynamics, as it is convenient to have the rotational equations of motion in the body frame, such that rotations about the center of the quadcopter can be expressed as:

$$
Q=I \dot{\omega}+\omega \times(I \omega)
$$

Then rewritten as:

$$
\dot{\omega}=\left[\begin{array}{c}
\dot{\omega}_{x} \\
\dot{\omega}_{y} \\
\dot{\omega}_{z}
\end{array}\right]=I^{-1}(Q-\omega \times(I \omega))
$$

Note: $I$ is the inertia matrix; $\omega$ is the angular velocity vector; $Q$ is the external torques vector.

Therefore acceleration of the quadcopter in its body frame is found by adding up the entire forces doing work on body surface. We then use the transformation matrix to convert the found accelerations into the body frame.

\section{SPFD-Technical Specifications}

\subsection{Sprays Copter Components [2]}

Figure 3 is an isometric view captured from the design modelers' graphics window of the Spray copters design.

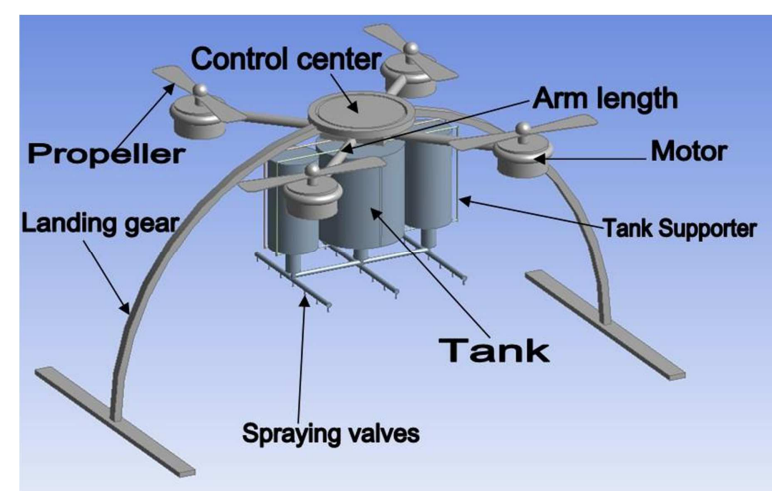

Figure 3. Sprays copter components.

Control center: Houses the control system and various electronics involved in keeping in perfect balance.

Arm length: Provides support to the Motor. 
Motor: Generates propulsion action via the driving of propellers.

Tank supporter: Holds the tank in position.

Tank: Storage for the liquid chemicals used to spray crops.

Spray valve device: Fabricated device for out letting of chemicals.

Landing gear: The base frame of the aircraft.

Propeller: Provides lift by the production of thrust via propulsive action.

\subsection{Frame Configuration \& Dimensions}

This is the stage at which think of in which configuration quadcopter will fly $[2,10]$, such as making the decision of which side will act as the front. There happens to be two types of frame configurations, the $\mathrm{X}$ configuration and the + configuration as Figure 4.

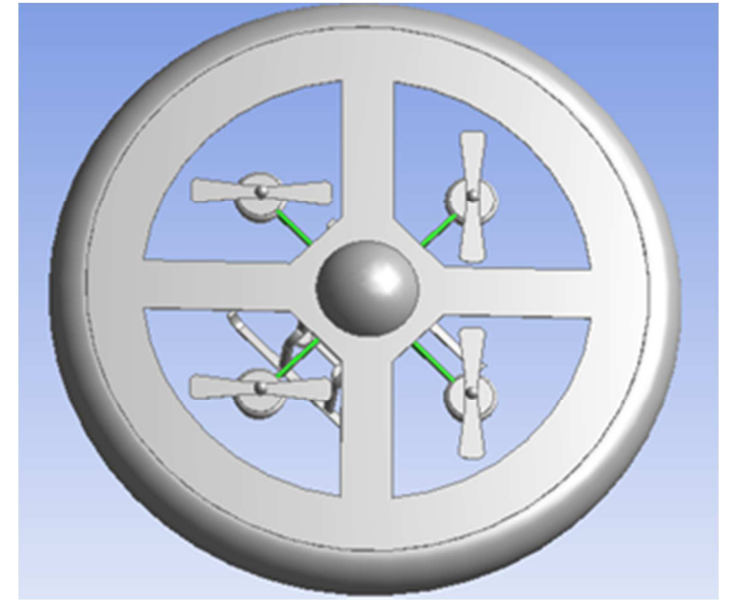

(a)

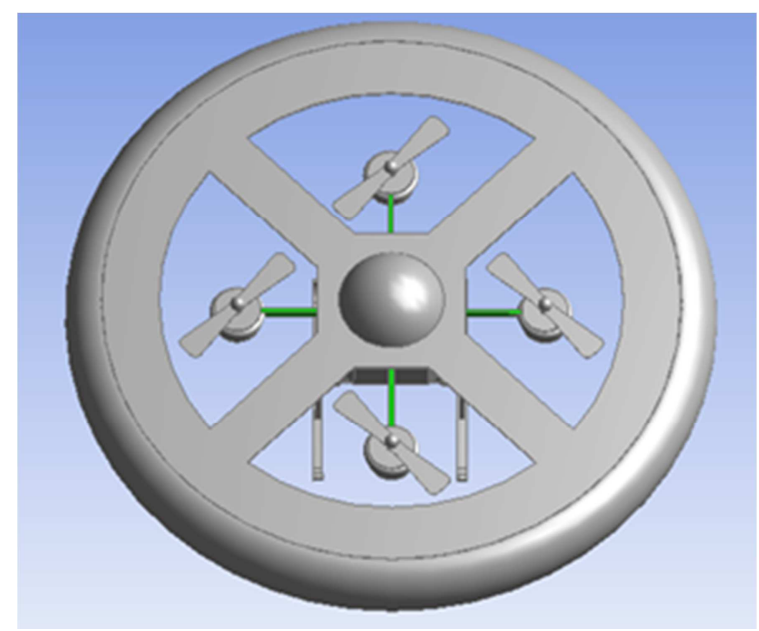

(b)

Figure 4. (a) X configuration; (b) + configuration.

We decided to go with the $\mathrm{X}$-configuration so that can have two motors working together on changing direction, unlike the + configuration which would only employ one motor. Structure views are shown in Figure 5.

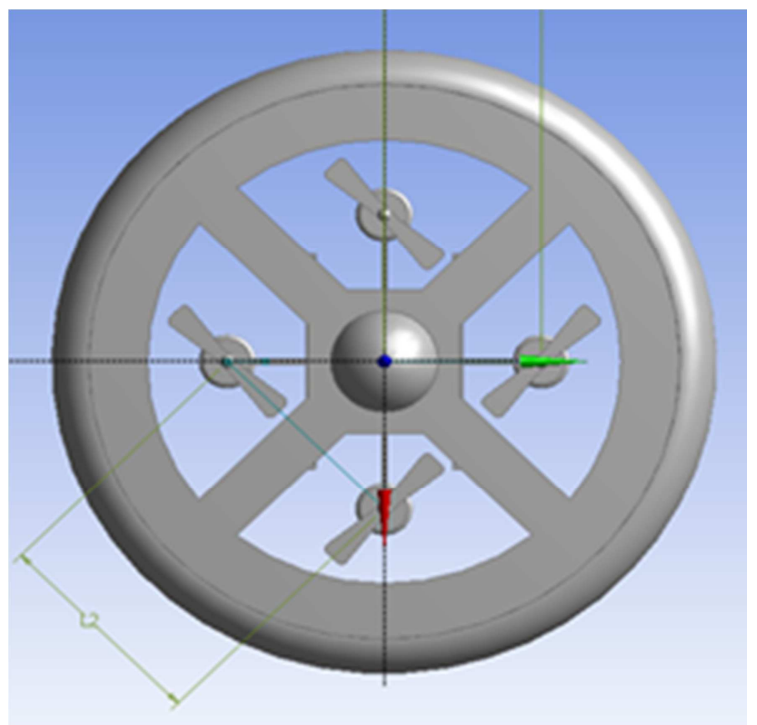

(a)

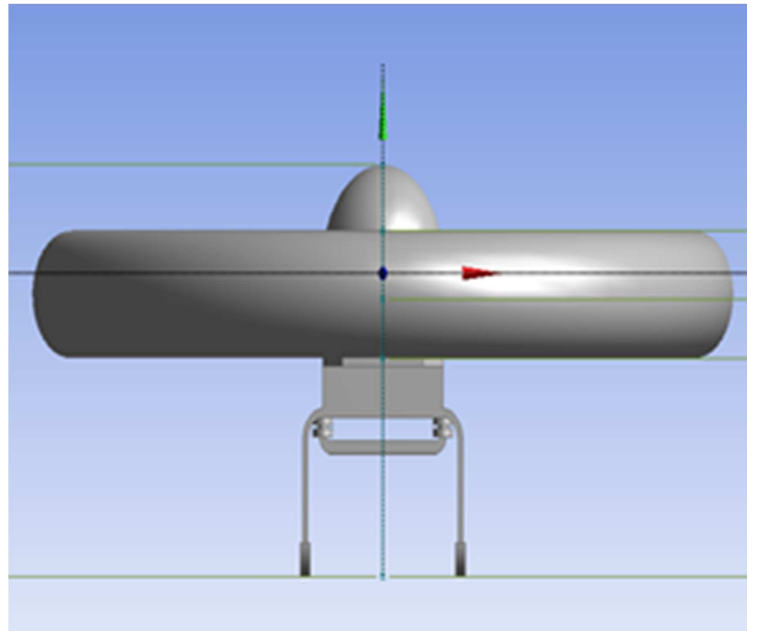

(b)

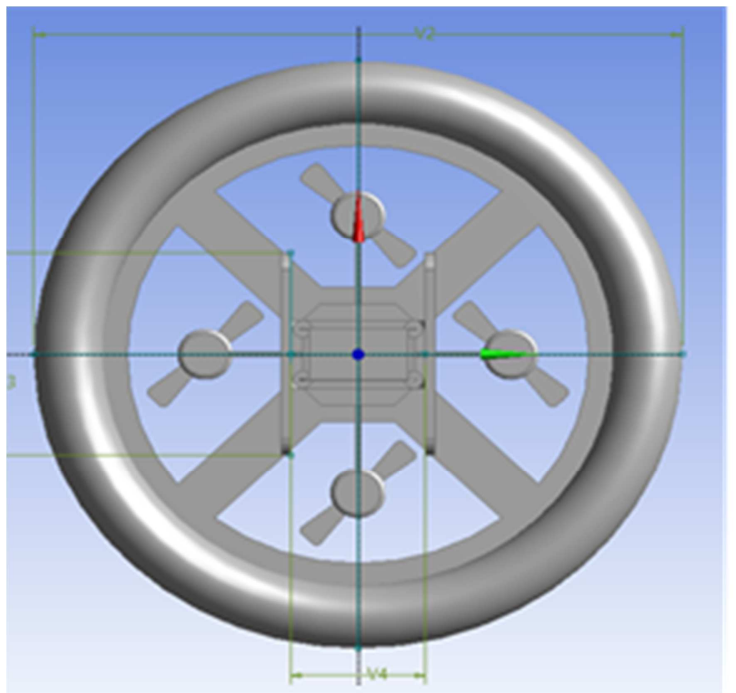

(c)

Figure 5. (a) Top View; (b) Front view; (c) Bottom view. 


\subsection{Flexible Solar Cell Configuration}

As shown in Figure 6, the SPFD quadcopter is designed to carry about 40 solar cells connected in series rated at $1.5 \mathrm{v}$ each, thus having 10 of these solar cells supplying $15 \mathrm{v}$ of the $14 \mathrm{v}$ needed to operate a single brushless motor, with attached power regulators to give protection to the motors.

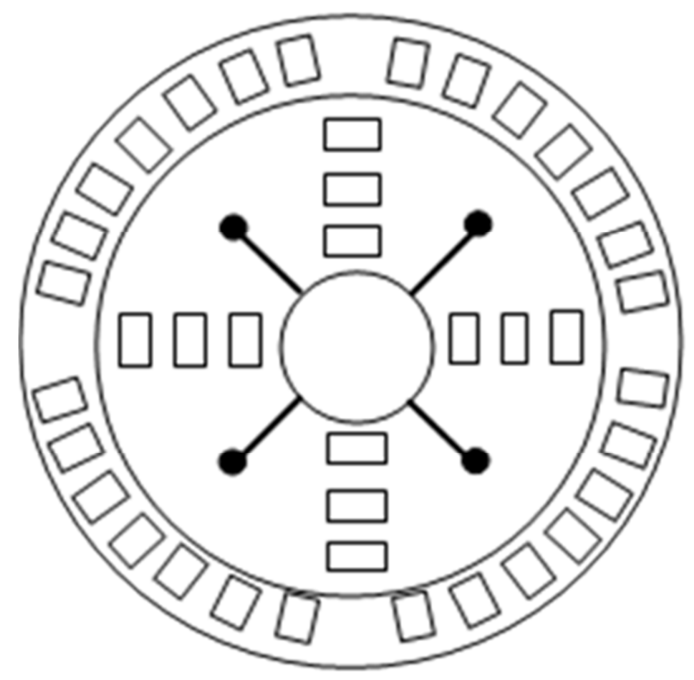

Figure 6. Distribution of solar panels.

\section{Components Compatibility Analysis}

A components compatibility theoretical analysis was carried for the electrical and mechanical devices via a flight estimation calculator, the $\mathrm{x}$-copter flight calculator developed by Markus Muller [10]. The motor characteristics at full throttle are shown in Figure 7.

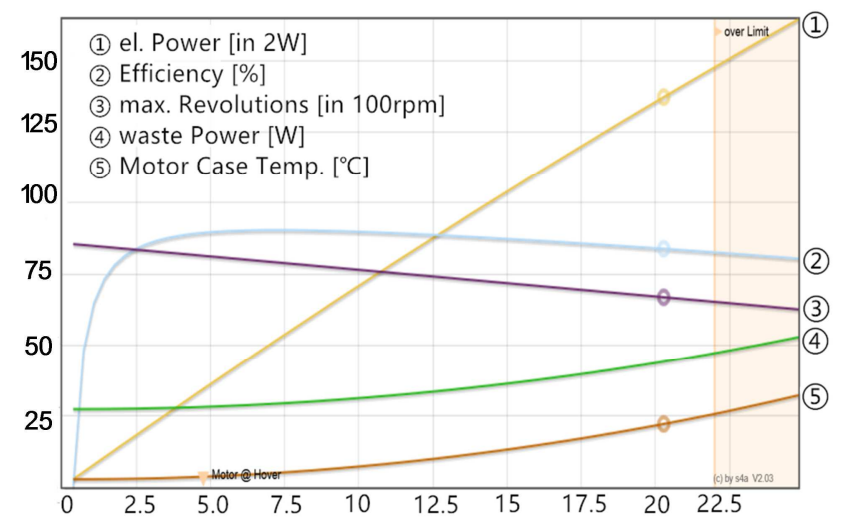

Figure 7. Motor characteristics at full throttle.

\subsection{Arm Deformation Analysis}

Table 1 shows the simulation setup data [11]. According to the SPDFs design, arm length is measured as the distance between the quad copters center of gravity and the rotors axis of rotation. During takeoff and flight courses the entire weight of the quad copter falls on the arms end sections attached to the control frame compartment resulting in a bending deformation of the arms that supports the motors as shown in the Figure 8 .

The red bolded arrow represents the entire models weight acting in the central section of the quadcopter which also happens to be the location of the center of gravity for the entire model. Such that a force is exerted in the central section as the motors work to provide lift, resulting into the deformation of the arm lengths [12].

Table 1. Simulation setup data.

\begin{tabular}{ll}
\hline Object Name & Solid \\
\hline State & Meshed \\
Coordinate System & Default Coordinate System \\
Reference Temperature & By Environment \\
Material & Aluminum Alloy \\
Length -X & $4 \mathrm{~mm}$ \\
Length -Y & $7 \mathrm{~mm}$ \\
Length -Z & $60 \mathrm{~mm}$ \\
Volume & $973.63 \mathrm{~mm}^{3}$ \\
Mass & $2.697 \mathrm{e}-003 \mathrm{~kg}$ \\
Nodes & 449928 \\
Elements & 264939 \\
Load (Y-component) & $1300 \mathrm{~N}(\mathrm{ramped})$ \\
Compressed Yield Strength & $280 \mathrm{MPa}$ \\
Tensile Yield Strength & $280 \mathrm{MPa}$ \\
Tensile Ultimate Strength & $310 \mathrm{MPa}$ \\
Young's Modulus & $71000 \mathrm{MPa}$ \\
Poisson's Ratio & 0.33 \\
Bulk Modulus & $6.9608 \mathrm{MPa}$ \\
Shear Modulus & $26692 \mathrm{MPa}$ \\
\hline
\end{tabular}

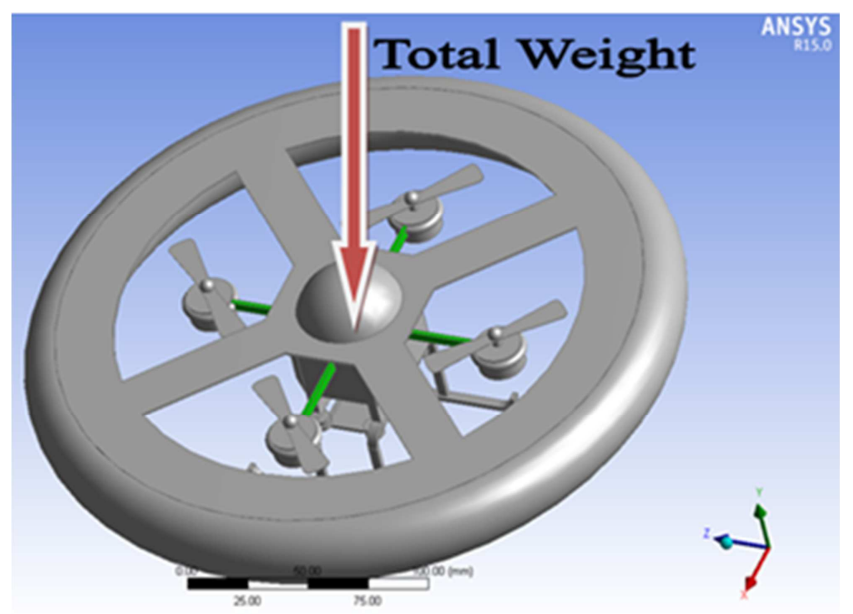

Figure 8. Force diagram for the quad copter.

As shown in Figure 9, a load of $1300 \mathrm{~N}$ is applied at the end section of the arm, exerting a downward force represented by a red bold arrow head pointing downwards. The other end of the arm is fixed to a surface such that when a force of $1300 \mathrm{~N}$ is applied on the other end, occurs a bending deformation along the stretched horizontal axis. The deformation process is shown by a photo shot of the run animation and graph simulation $[2,13]$. 


\subsection{Total Deformation}

Figure 10 shows the deformation process along the arm length represented by coded colors of dark blue to red with an animation time frame from 0 to 1 .

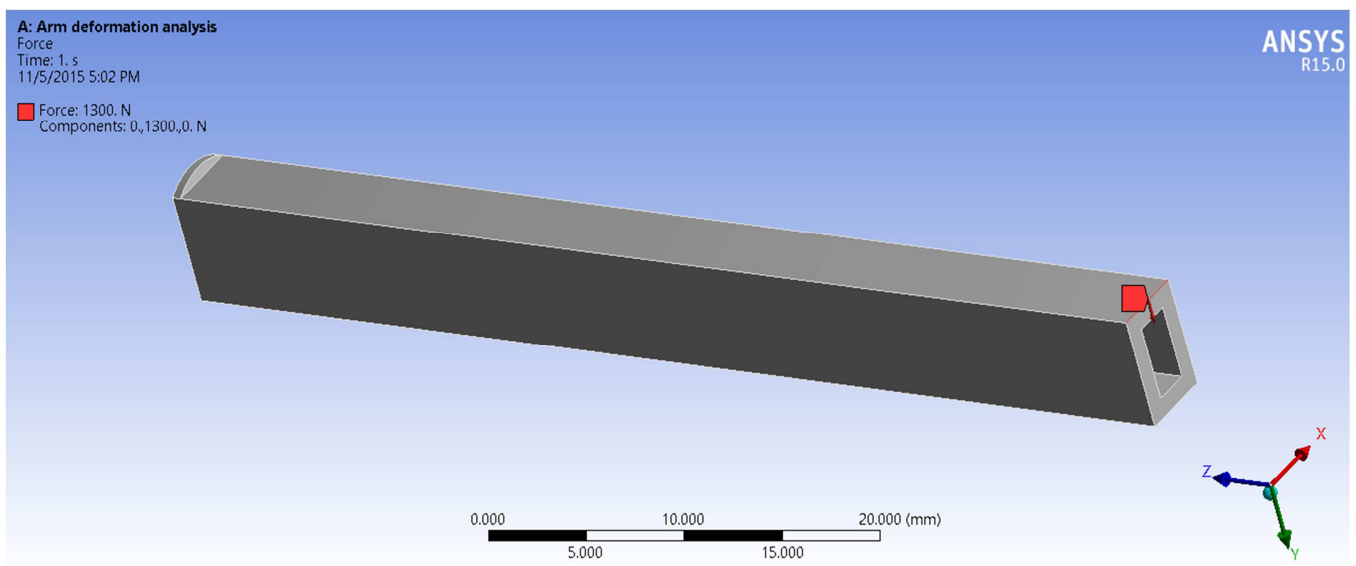

Figure 9. A load applied at the end section of the arm.

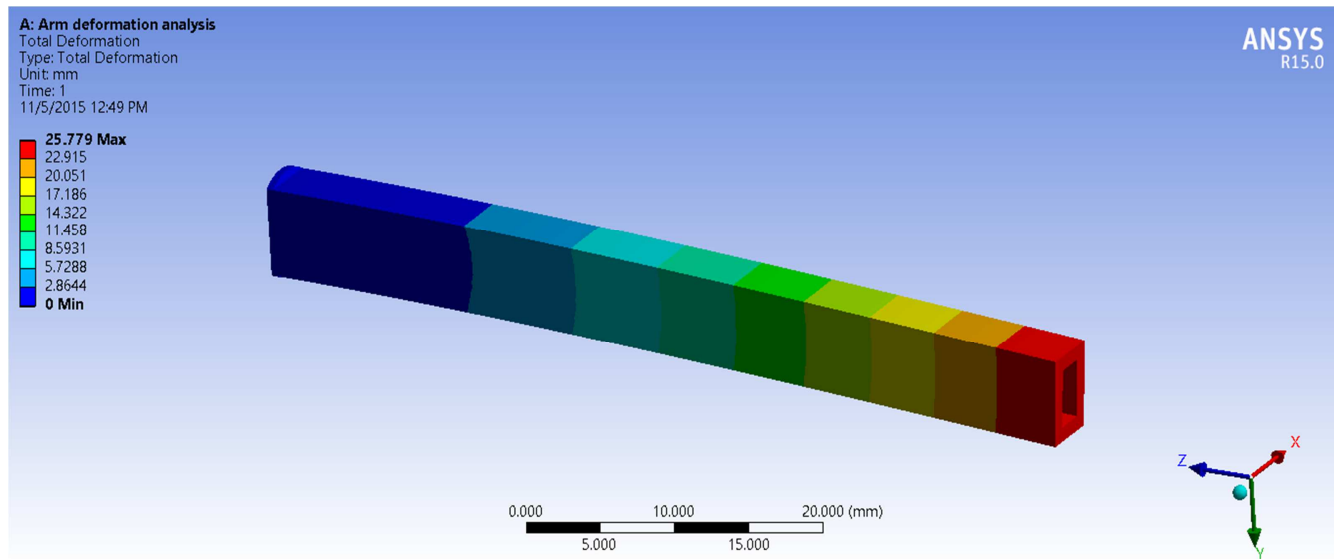

Figure 10. The deformation process of the arm.

\subsection{Equivalent Stress}

As shown in Figure 11 as the motor drives the propellers, such that thrust is produced by the propellers to provide lift for the quadcopter, the arm length supporting the motors and the models body experiences both tensile and compression stresses, and due to the magnitude of the stresses the arm section suffers strain effects. The fixed end sections show symptoms of fatigue which results to material part failure as initial cracks tend to develop which leads to slits or splits of the arm material or from an attached member, in this case the motor manifold or the electronics compartment frame on the other end [2].

\subsection{Normal Stress (X-axis)}

During the course of conducting a normal stress (x-axis) analysis of the arm length, it was found that when deformation reaches a maximum value of about $25873 \mathrm{MPa}$ represented in red color, it so happens that the part shows signs of fatigue failure at the end section of the top surface normal to the $\mathrm{X}$-axis as shown in Figure12.

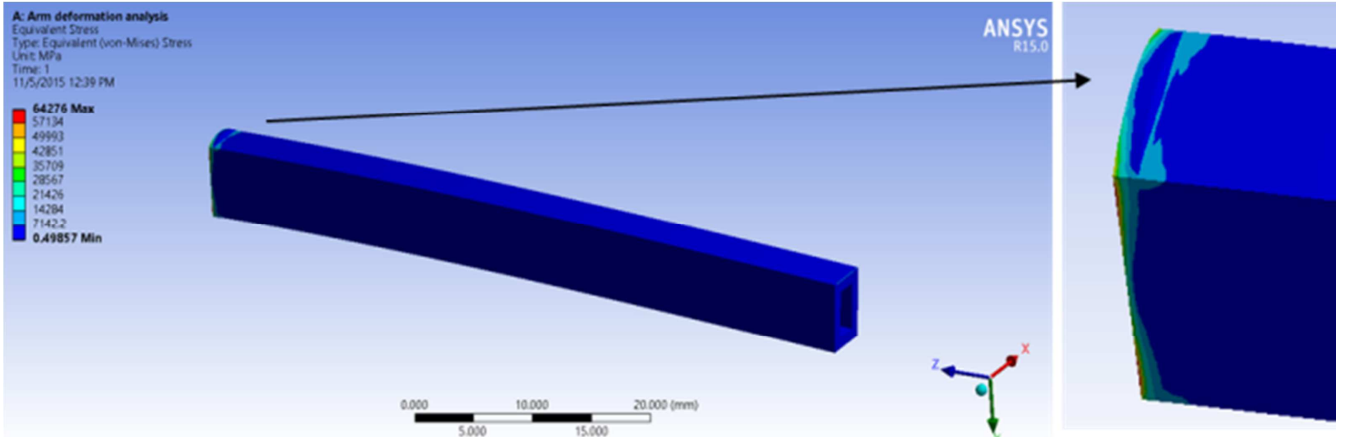

Figure 11. Results of the stress distribution in the arm. 


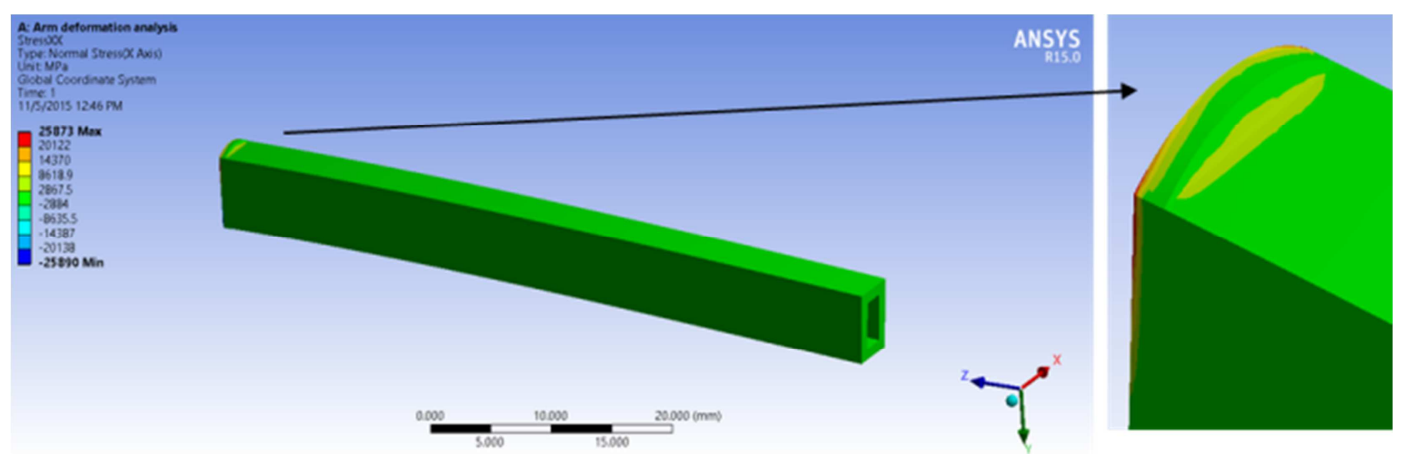

Figure 12. The strength of the top of the arm.

\subsection{Probable Crack Formation Locations}

The critical location analysis shows how fatigue lives can be calculated from a sequence of local strains and shows how stress concentration factors can be used to calculate fatigue lives for components which contain notches or about to develop notches like in the Figure 13 depicting the affected area.

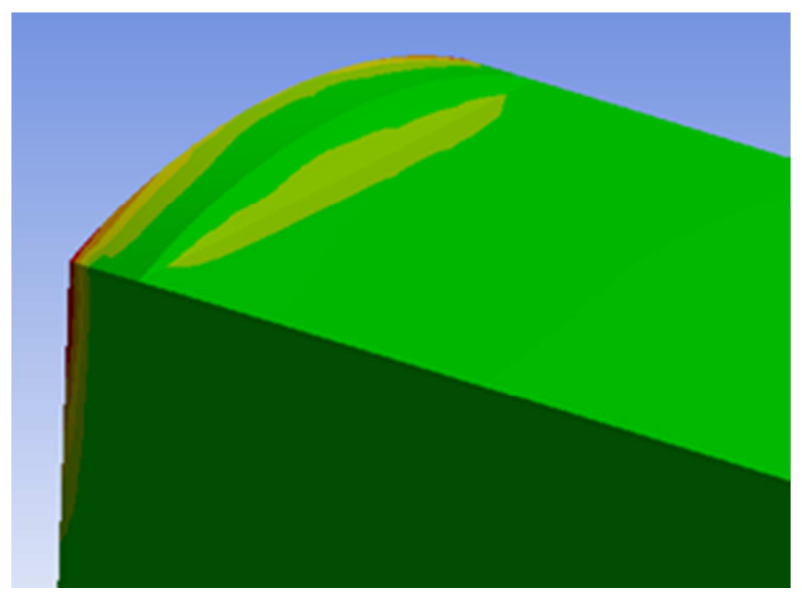

Figure 13. Probable crack formation locations.

As the developed crack length increases, the strength of the structure reduces to a level that it fails to withstand normal service loads. Knowledge of fracture mechanics helps the designer to calculate the crack size that can be allowed at the expected service load, the residual strength as a function of crack size, the duration it takes the crack to grow into a critical size, the period between the inspection of the cracks and also the size of initial flaws tolerable in a new component $[2,13]$.

\section{The Analysis of Body Deformation}

The shape of the arm is a square column with a long length. The arm, with some connecting holes, supports the plane of the ring plate. All the solar panels are distributed on the ring plate, and the ring plate also exerts pressure on the arm. Therefore, the deformation of the arm also affects the shape of the ring plate and the working of the solar cell $[2,14]$.

In addition, the SPFD quadcopter has different load types in different working conditions (windless, windy, hovering, etc). The laying process and layering of the material can affect the $\mathrm{UAV}$, especially the strength and rigidity of the arm, causing different degrees of arm deformation. In this regard, it is necessary to conduct special research in the follow-up work, and will not be repeated here.

\section{Conclusion}

In conclusion, we have tested utilizing the usefulness of the Ansys designing tool software and also experimented with solar panels in collaboration with an MPPT for battery charging purposes. The quadcopter has been designed to be powered by 40 solar thin film cells rated at $1.5 \mathrm{v}$ per panel. A fatigue analysis is conducted by use of the "ANSYS" designing and simulating program shows the level of deformation in some sections of the model.

\section{Acknowledgements}

This paper is one of the periodic achievements of the National Natural Science Foundation of China (Grant No. 51365042) and The Project Sponsored by the Scientific Research Foundation for the Returned Overseas Chinese Scholars, State Education Jiangxi (Grant No. DB201406147). The authors would like to thanks for them.

\section{References}

[1] Y. Li, S. Gao, A. K. Kan, L. Zhang. Design of an UAV search and rescue system with the ECG monitor function [J]. Chinese High Technology Letters, vol. 25 (Z2), 2015, pp. 941-947.

[2] Mwanza Taipa Raymond. Spray-Copter Design and Manufacture [D]. Nanchang Hangkong University Master's thesis. 2016. 03. pp. 1-10

[3] Ying XU, Xiao Ping HU, Ben Shuang ZHANG, Jian. The Design and Dynamics Analysis of Robot Drilling Platform Special for Cutting of Hardworking Material [C]. The 2015 International Conference on Mechanical Science and Mechanical Design (MSMD2015), Changsha, China, 2015. 12, $43-49$

[4] Long LI, Ying XU, Miao SUN, Huang HUANG, Airfoil Optimization Design of Flying Wing Configuration [C], The 2015 International Conference on Mechanical Science and Mechanical Design [C]. The 2015 International Conference on Mechanical Science and Mechanical Design (MSMD2015), Changsha, China, 2015. 12.418-424. 
[5] M. M. Abdelrahman, M. M. Elnomrossy and M. R. Ahmed, Development of Mini Unmanned Air Vehicles [C]. 13th International Conference on Aerospace Sciences \& Aviation Technology, ASAT- 13, Cairo, Egypt, May 2009, pp1-13.

[6] C. ZHOU, X. Y. XIANG, X. ZHONG, Y. HE. Application and prospect of unmanned aerial vehicle in agriculture $[\mathrm{J}]$. Hunan Agricultural Sciences [J]., vol. (11), 2017, pp. 80-82+86.

[7] X. S. Feng. Overview on application of solar unmanned aerial vehicle [J]. Solar Energy, vol. (07), 2018, pp. 10-15+75.

[8] L. Shen, Z. Z. Liu. Solar quad-rotor UAV design and implementation [J]. Automation \& Instrumentation, vol. (03), 2017, pp. 51-54.

[9] Z. D. Li, H. S. Liu. Modeling and control simulation of quadrotor [J] Automation Application, vol. (09), 2017, pp. $57-59+150$.
[10] P. Y. Feng. Quadrotor UAV modeling and PID controller design [J] Industrial Design, vol. (06), 2018, pp. 135-137.

[11] Q. H. YANG, Z. Q. SONG, L. SHI. Somatosensory control of quadrotor UAV based on Kinect [J] Journal of Naval Aeronautical and Astronautical University, vol. 24 (05), 2009, pp. 499-502.

[12] H. LI, L. B. LU, G. D. JIN. Modeling and flight control of quadrotor [J]. Transducer and Microsystem Technologies, vol. 34 (08), 2015, pp. 99-102.

[13] G. L. Gao, Z. K. Li, B. F. Song, X. Ding. Key technologies of solar powered unmanned air vehicle [J]. Flight Dynamics, vol. 28 (01), 2010, pp. 1-4.

[14] Y. GUO, D. W. WANG, Y. DENG. Modeling and flight control of quadrotor $[\mathrm{J}]$. Transducer and Microsystem Technologies, vol. 36 (11), 2017, pp. 38-41. 